\title{
Basis risk in static versus dynamic longevity-risk hedging
}

This is the peer reviewed version of the following article:

Original:

De Rosa, C., Luciano, E., Regis, L. (2017). Basis risk in static versus dynamic longevity-risk hedging. SCANDINAVIAN ACTUARIAL JOURNAL, 17(4), 343-365 [10.1080/03461238.2015.1134636].

Availability:

This version is availablehttp://hdl.handle.net/11365/1001674

since 2018-11-02T18:35:35Z

\section{Published:}

DOI:10.1080/03461238.2015.1134636

Terms of use:

Open Access

The terms and conditions for the reuse of this version of the manuscript are specified in the publishing policy. Works made available under a Creative Commons license can be used according to the terms and conditions of said license.

For all terms of use and more information see the publisher's website.

(Article begins on next page) 


\title{
Basis risk in static versus dynamic longevity-risk hedging*
}

\author{
Clemente De Rosa ${ }^{\dagger}$ Elisa Luciano ${ }^{\ddagger}$ Luca Regis ${ }^{\S}$
}

October 19, 2015

\begin{abstract}
This paper provides a tractable, parsimonious model for assessing basis risk in longevity and its effect on the hedging strategies of Pension Funds and annuity providers. Basis risk is captured by a single parameter, that measures the co-movement between the portfolio and the reference population's longevity. The paper sets out the static, full and customized swap-hedge for an annuity, and compares it with a dynamic, partial and index-based hedge. We calibrate our model to the UK and Scottish populations. The effectiveness of static versus dynamic strategies depends on the rebalancing frequency of the second, on the relative costs, and on basis risk, which does not affect fully-customized, static hedges. We show that appropriately calibrated dynamic hedging strategies can still be reasonably effective, even at low rebalancing frequencies.
\end{abstract}

Keywords: longevity risk, customized vs. indexed hedge, longevity swaps, longevity bonds, rebalancing frequency.

JEL classification: G22, G32.

${ }^{*}$ The paper has been published in the Scandinavian Actuarial Journal, Volume 4, 2017, pp. 343-365. The Authors would like to thank conference participants to the IRMC Conference 2015, the 19th IME Conference, the Longevity 11 Conference. The Authors gratefully acknowledge financial support by the Global Risk Institute, Canada.

${ }^{\dagger}$ Collegio Carlo Alberto, clemente.derosa@carloalberto.org, Via Real Collegio 30, 10024 Moncalieri, Italy, +390116705228.

${ }^{\ddagger}$ Corresponding Author. University of Torino, Collegio Carlo Alberto and Netspar; ESOMAS Department, Corso Unione Sovietica 218/bis, 10134, Torino, Italy; e-mail: elisa.luciano@unito.it; phone number: + 390116705230 (5200).

${ }^{\S}$ IMT Institute for Advanced Studies Lucca and Collegio Carlo Alberto; AXES Research Unit - Piazza San Francesco 19, 55100 Lucca, Italy; e-mail: luca.regis@imtlucca.it.; phone number: +3905834326721 . 


\section{Introduction}

Pension funds and life insurance companies providing annuities are affected by the so-called longevity risk, which is the risk that people live longer than expected when the fund or company priced and reserved their policies. So, while increasing longevity is welcome from the social point of view, it is one of the risks that annuity providers - which we will call insurers for brevity - have to face. Being a systematic risk, it cannot be diversified away by increasing the size of portfolios. Still, insurers can hedge it, using mortality derivatives, which were first proposed by Blake and Burrows (2001). So far, such derivatives have been most frequently traded over-the-counter (OTC) and written on the insurer's specific policyholders' population. So, they are customized to fulfil the needs of the insurer. Alongside these deals, a market for standardized products has slowly emerged. These products leave the insurance company exposed to so-called basis risk, because they are written on a reference population that does not coincide with the insured one. In the surge of markets for longevity derivatives, the debate on the pros and cons of fully customized versus index-based products is very much open. In practice, actors seeking coverage with a fully customized OTC product build a full, static hedge, such as an s-forward or a longevity swap, while not fully customized derivatives (non-OTC for brevity) provide a partial, dynamic hedge. ${ }^{1}$ In the first case, the hedging strategy is not adjusted over time. In the second case, coverage calls for adjustment over time. In the absence of basis risk, dynamic strategies would converge to a full, instead of a partial or approximated coverage, if rebalancing would be continuous. With basis risk, they do not converge. In both cases, they "cost", in the sense that - to make them self-financing - the insurer must fund them through a "bank account", whose final value is the hedging cost. So, while static hedges have an initially defined and considerable cost, dynamic strategies have a cost, whose amount depends on the rebalancing technique, frequency and the actual path of the underlying insurance contracts. All in all, dynamic strategies are partial, leave the insurer exposed to basis risk, but have potentially smaller costs than static hedges.

Against this background, the aim of our paper is to propose a simple framework for evaluating the effectiveness of static versus dynamic longevityhedging solutions, in the presence of basis risk. Our first original contribution consists in providing a simple model for basis risk in longevity, by separating forecast errors that are common to the general and the insured population from idiosyncratic errors, which are proper of the latter population. Longevity risk is represented through a stochastic mortality approach, namely a mortality intensity which is itself a stochastic process. We model

\footnotetext{
${ }^{1}$ For a description of a customized and an index-based transaction involving JP Morgan as a buyer of longevity risk, see Barrieu et al. (2012).
} 
basis risk by assuming that the intensity of the general population is imperfectly correlated with the insured one. So, we encapsulate basis risk in a single, comovement parameter.

Once basis risk is modeled, our second important contribution consists in providing fair prices of static and dynamic hedging instruments in closed form. Our static hedge entails the use of an OTC longevity swap written on the portfolio population, thereby entailing no basis risk. We consider dynamic hedges that approximate the hedge up to the first (Delta) or second (Delta-Gamma) or time (Theta) change in the longevity forecast error, and are based on index-related longevity bonds.

Third, we assess the relative effectiveness of static versus dynamic strategies in a calibrated environment, where the portfolio population is made by 65 -year-old Scottish males and the reference is made by 65 -year-old UK males. The dynamic strategy gives rise to two types of error or costs, that we disentangle: the error due to its approximated nature, together with discretetime rebalancing (as in Dahl et al., 2011), and the error due to unhedgeable basis risk. Since these errors are stochastic, we measure them through their 99.5 percentile of the bank account which makes the strategy self-financing. This percentile is the greatest cost that the index-based hedge may entail, in $99.5 \%$ of the possible cases. We determine the cost of the static hedge which - split along the life of the swap as a fee - equates the given percentile, and makes the two strategies cost the same. Beyond this maximum fee, the dynamic hedge dominates the static hedge.

To anticipate on our results, we show that when basis risk is null, the fee of the non-OTC strategy stays between $0.01 \%$ and $0.06 \%$. In this case, the cost of dynamic hedges is due only to their partial nature, and to discrete hedging. Theta hedge does not improve much the effectiveness of a Delta-Gamma strategy. When there is basis risk and the dynamic hedge is performed using a Delta-Gamma-Theta strategy the fee ranges between $0.12 \%$ and $0.41 \%$, depending on the rebalancing frequency, which stays between a quarter and one year. Comparing the two fees, with and without basis risk, we can measure the impact of basis risk. Even if the fees we computed are relatively low, the one when basis risk is present is around seven to twelve times the other, depending on the rebalancing frequency. It would be much bigger for more infrequent rebalancing and with other, less precise dynamic hedges. We conclude that basis risk might be relevant for annuity providers' hedges, but hedging strategies, if appropriately designed and calibrated, can still be very effective.

The paper unfolds as follows: in Section 2 we review some related literature, in Section 3 we set up the model for longevity and financial risk evaluation, in Section 4 we introduce basis risk. In Section 5 we present the liabilities to be hedged. In Sections 6 and 7 we present static and dynamic hedges. In Section 8 we implement the strategies on the UK-Scottish male population aged 65 , both without and with basis risk, and compare their 
effectiveness. The last Section summarizes and concludes.

\section{Related Literature}

The effectiveness of static hedging strategies in the presence of basis risk has been examined by Ngai and Sherris (2011). Comparing different cash-flow matching hedging strategies achieved using various instruments, such as qforwards, longevity bonds or swaps, they show that static hedging is effective in reducing tail risk (expected shortfall) and that the impact of basis risk on effectiveness is limited. In Coughlan et al. (2011), the authors argue that, due to the lack of a proper framework to understand Basis Risk, there has been a widely diffused misconception that index-based longevity hedging is ineffective. So, they describe a framework to evaluate basis risk and to determine the effectiveness of longevity hedging when standardized index-based instruments are used. In their case study, using bootstrap from historical data, they find that, despite the presence of basis risk, if the correlation between the dynamics of the reference population underlying the hedging instrument and the liability portfolio one is high, the hedge remains very effective. The importance of considering basis risk is highlighted by Cairns et al. (2014), who, using simulated rather than bootstrapped data, show that population basis risk is the most important determinant of the hedge effectiveness of a static value-hedge longevity swap. Haberman et al. (2014) also point out the importance of assessing basis risk in indexed solutions, in order to understand the residual risk left, also in the light of Solvency II capital requirements. In their paper, they provide a comprehensive treatment of basis risk measurement, addressing the issues of model selection and proper calibration.

Li and Hardy (2011) compare four different extensions of the Lee-Carter model, suitable to model basis risk. The analysis identifies the Augmented Common Factor model as most appropriate among those tested. Such model assumes, similarly to the model we propose in this paper, that the mortalities of two populations depend on a common and on a population-specific factor. Application to the hedging of a Canadian pension plan exposure to longevity risk using a portfolio of $q$-forwards indexed to the US population shows that a static longevity hedge can still be reasonably effective even when basis risk is present.

Continuous-time modeling and dynamic hedging of longevity basis risk, as in our paper, are tackled by Dahl et al. (2008) and Wong et al. (2014). The former consider two populations and model mortality improvements using two mean-reverting CIR processes driven by a bi-dimensional standard Brownian Motion. In this setting, the paper provides risk-minimizing strategies of longevity risk by dynamically trading in a survivor swap when basis risk is present. Wong et al. (2014) derive the mean-variance hedging strategy 
for longevity risk using an instrument contingent on a mortality index, where the mortality intensity of the insurer's portfolio population is correlated and cointegrated to the mortality index itself. Differently from these two papers, our work focuses on local rather than global hedging and provides a calibrated assessment of the impact of basis risk and hedging frequency on the effectiveness of dynamic strategies.

\section{Longevity and interest rate risk modeling}

We model longevity risk assuming that the time to death of an individual belonging to a specific generation follows a Poisson process with stochastic intensity. We consider a standard filtered probability space $(\Omega, \mathcal{F}, \mathbb{Q})$ satisfying the usual assumptions and on which a filtration $\mathcal{F}_{t}$ is defined. The measure $\mathbb{Q}$ is the so-called risk-neutral measure. Below, we will discuss the relationship between this measure and the historical one.

The mortality intensity of a specific generation is described by a so-called Cox-Ingersoll and Ross (CIR) process, i.e. a Feller process of the type:

$$
d \lambda(t)=(a+b \lambda(t)) d t+\sigma \sqrt{\lambda(t)} d W(t),
$$

with $a>0, b>0, \sigma>0, \lambda(0)=\lambda_{0} \in \mathbb{R}^{++}$. The reason behind the assumption $b>0$ is that the process is expected to have no mean reversion. The previous SDE describes the evolution (for a given generation) of the intensity of mortality arrival over calendar time. Because the generation ages over time, the drift simply ensures that the expected change in the intensity is affine and increasing in the intensity itself.

If the initial point $\lambda_{0}$ is strictly positive and the coefficients satisfy the following condition:

$$
a \geq \frac{\sigma^{2}}{2},
$$

then the mortality intensity $\lambda(t)$ is strictly positive for every $t$, almost surely. Hence, to obtain a satisfactory calibrated model for the intensity process, we impose this condition on the parameters in the calibration procedure.

Consistently, we assume that the spot interest rate - or interest rate intensity - follows a CIR process of the type:

$$
d r(t)=(\bar{a}-\bar{b} r(t)) d t+\bar{\sigma} \sqrt{r(t)} d W^{\prime}(t),
$$

with $\bar{a}>0, \bar{b}>0, \bar{\sigma}>0, r(0)=r_{0} \in \mathbb{R}^{++}$, where the Wiener process $W^{\prime}$ is independent of $W 2^{2}$ This last assumption entails independence between longevity and interest rate risks. The negative sign preceding $\bar{b}$ and its strict positivity guarantee that the process for the interest-rate incorporates mean

\footnotetext{
${ }^{2}$ Let the filtration $\mathcal{F}_{t}$ be the filtration generated by the two Brownian motions.
} 
reversion, which is a usual assumption in the interest-rate domain. The coefficient $\bar{b}$ is called speed of mean reversion and represents the speed at which the short rate $r(t)$ returns to its long-run value $\bar{a}$.

Similarly to the longevity case, the restriction on the parameters that, together with the positivity of the initial point $r_{0}$, guarantees that the interest rate $r(t)$ never turns negative is

$$
\bar{a} \geq \frac{\bar{\sigma}^{2}}{2}
$$

At each point in time, the conditional distributions of the mortality intensity and the interest rate are given, up to a scale factor, by a noncentral chi-square distribution ${ }^{3}$

Denoting as $\tau$ the time to death, the conditional survival probability from $t$ to $T$ is

$$
S(t, T)=\mathbb{P}(\tau \geqslant T \mid \tau>t) .
$$

To proceed to the pricing and hedging of insurance products, the risk-neutral dynamics of the two previous processes is needed. However, for calibration purposes, its effective or historical version may be the one stemming from the data, at least for the longevity case. In order to keep the notation simple, we assume that there is no risk premium in the longevity market or, equivalently, that equation (1) holds under both measures. Therefore, the

\footnotetext{
${ }^{3}$ In details, given two time instants $u<t$, then the distribution of $\lambda(t)$ conditional on $\lambda(u)$ is

$$
\lambda(t) \approx \frac{\sigma^{2}\left(e^{b(t-u)}-1\right)}{4 b} \mathcal{X}_{d}^{\prime 2}(\nu),
$$

where $\mathcal{X}_{d}^{\prime 2}(\nu)$ denotes the density of a noncentral chi-square random variable with $d$ degrees of freedom, where

$$
d=\frac{4 a}{\sigma^{2}}
$$

and the noncentrality parameter $\nu$ is

$$
\nu=\frac{4 b e^{b(t-u)}}{\sigma^{2}\left(e^{b(t-u)}-1\right)} \lambda(u)
$$

Similarly, the distribution of $r(t)$ conditional on $r(u)$ is

$$
r(t) \approx \frac{\bar{\sigma}^{2}\left(1-e^{-\bar{b}(t-u)}\right)}{4 \bar{b}} \mathcal{X}_{\bar{d}}^{\prime 2}(\bar{\nu})
$$

where $\mathcal{X}_{\bar{d}}^{\prime 2}(\bar{\nu})$ denotes the density of a noncentral chi-square random variable with $\bar{d}$ degrees of freedom, with

$$
\bar{d}=\frac{4 \bar{a}}{\bar{\sigma}^{2}}
$$

and the noncentrality parameter $\nu$ is

$$
\bar{\nu}=\frac{4 \bar{b} e^{-\bar{b}(t-u)}}{\bar{\sigma}^{2}\left(1-e^{-\bar{b}(t-u)}\right)} r(u) .
$$


calibration of the longevity intensity is performed by estimating its dynamics under the historical measure and, then, using it also under the risk-neutral measure. The calibration of the interest rate dynamics is, on the other hand, performed directly under the risk-neutral measure, thus incorporating the risk premium. As a consequence, the conditional survival probability 11) can be represented as an expectation under the risk neutral measure:

$$
S(t, T)=\mathbb{E}^{\mathbb{Q}}\left[\exp \left(-\int_{t}^{T} \lambda_{x}(s) d s\right) \mid \mathcal{F}_{t}\right] .
$$

Given our model choice, this expectation becomes:

$$
S(t, T)=A(t, T) e^{-B(t, T) \lambda(t)},
$$

where $A(t, T)$ and $B(t, T)$ solve an appropriate system of Riccati equations, being

$$
\begin{aligned}
& A(t, T)=A(t, T ; a, b, \sigma)=\left(\frac{2 \gamma e^{\frac{1}{2}(\gamma-b)(T-t)}}{(\gamma-b)\left(e^{\gamma(T-t)}-1\right)+2 \gamma}\right)^{\frac{2 a}{\sigma^{2}}}, \\
& B(t, T)=B(t, T ; a, b, \sigma)=\frac{2\left(e^{\gamma(T-t)}-1\right)}{(\gamma-b)\left(e^{\gamma(T-t)}-1\right)+2 \gamma}
\end{aligned}
$$

where $\gamma=\sqrt{b^{2}+2 \sigma^{2}}$. As shown in Fung et al. (2014), the above specification guarantees also that the limit of the survival probability, when $T$ diverges, is zero.

For any given $t$, it is possible to compute the log-derivative of the survival probability, which is referred to as the "forward" mortality intensity for time $T$, since it represents its forecast at time $t$. By definition ${ }^{4}$

$$
f(t, T)=-\frac{\partial \ln S(t, T)}{\partial T}=-\frac{\partial \ln A(t, T)}{\partial T}+\frac{\partial B(t, T)}{\partial T} \lambda(t),
$$

Using a technique described in Jarrow and Turnbull (1994) and Luciano et al. (2012), which exploits the definition of "forward" intensity, we can write the survival a 5

$$
S(t, T)=e^{-X(t, T) I(t)+Y(t, T)},
$$

$$
\begin{aligned}
& { }^{4} \text { It is easy to show that } \\
& \qquad \begin{aligned}
\frac{\partial \ln A(t, T)}{\partial T} & =\frac{2 a}{\sigma^{2}}\left[\frac{1}{2}(\gamma-b)-\frac{\gamma e^{\gamma(T-t)}}{e^{\gamma(T-t)}-1+\frac{2 \gamma}{\gamma-b}}\right], \\
\frac{\partial B(t, T)}{\partial T} & =\frac{4 \gamma^{2} e^{\gamma(T-t)}}{\left[(\gamma-b)\left(e^{\gamma(T-t)}-1\right)+2 \gamma\right]^{2}} .
\end{aligned}
\end{aligned}
$$

${ }^{5}$ Using the fact that $\lambda(t)=I(t)+f(0, t), 20$ becomes

$$
S(t, T)=A(t, T) \exp \left(-B(t, T)\left[I(t)-\left.\frac{\partial \ln A(t, T)}{\partial T}\right|_{(0, t)}+\left.\lambda(0) \frac{\partial B(t, T)}{\partial T}\right|_{(0, t)}\right]\right) .
$$

Hence, we have an expression for the survival equivalent to 13 . 
where $I(t)=\lambda(t)-f(0, t)$ and $X(t, T)$ and $Y(t, T)$ are deterministic functions of the parameters of the intensity process and of time $t$ and $T$ :

$$
\begin{aligned}
X(t, T) & =B(t, T) \\
Y(t, T) & =\ln A(t, T)-B(t, T)\left[-\left.\frac{\partial \ln A(t, T)}{\partial T}\right|_{(0, t)}+\left.\lambda(0) \frac{\partial B(t, T)}{\partial T}\right|_{(0, t)}\right] .
\end{aligned}
$$

The term $I$ is called longevity risk factor. It is the difference between the actual intensity at time $t$ and its forecast made at time 0. Expression (20) will play a crucial role in hedging, because it encapsulates all riskiness in the $I$ factor, which has the intuitively nice interpretation of a forecast error.6

The discount factor or bond price for time $t$, under any stochastic process for the spot rate, is

$$
D(t, T)=\mathbb{E}\left[\exp \left(-\int_{t}^{T} r(u) d u\right) \mid \mathcal{F}_{t}\right]
$$

which, in the CIR case, becomes

$$
D(t, T)=\bar{A}(t, T) e^{-\bar{B}(t, T) r(t)},
$$

where $\bar{A}(t, T)=A(t, T ; \bar{a},-\bar{b}, \bar{\sigma})$ and $\bar{B}(t, T)=B(t, T ; \bar{a},-\bar{b}, \bar{\sigma})$ and $\bar{\gamma}=$ $\sqrt{\bar{b}^{2}+2 \bar{\sigma}^{2}}$. As in the longevity case, the bond value can be reformulated as

$$
D(t, T)=e^{-\bar{X}(t, T) J(t)+\bar{Y}(t, T)},
$$

where

$$
\begin{aligned}
\bar{X}(t, T) & =\bar{B}(t, T) \\
\bar{Y}(t, T) & =\ln \bar{A}(t, T)-\bar{B}(t, T)\left[-\left.\frac{\partial \ln \bar{A}(t, T)}{\partial T}\right|_{(0, t)}+\left.r(0) \frac{\partial \bar{B}(t, T)}{\partial T}\right|_{(0, t)}\right]
\end{aligned}
$$

and $J$ is the financial risk factor, measured by the difference between the short and forward rate:

$$
J(t)=r(t)-F(0, t)
$$

The forward rate $F(t, T)$ represents the fair price at time $t$ for a forward contract on the spot rate at $T$. It is computed, similarly to the forward

\footnotetext{
${ }^{6}$ As we know, the forecast error in longevity has been substantial over the last decades. Approximately, expected lifetime improvement has been underestimated by 3 years over the last century (see International Monetary Fund (IMF) (2012)).
} 
mortality intensity, as ${ }^{7}$

$$
F(t, T)=-\frac{\partial \ln D(t, T)}{\partial T}=-\frac{\partial \ln \bar{A}(t, T)}{\partial T}+\frac{\partial \bar{B}(t, T)}{\partial T} r(t),
$$

So, also in the bond case, the reformulation in terms of the risk factor allows us to synthesize in a unique spread the forecast error that economic agents can make and that they may be willing to hedge.

\section{Basis risk}

Up to now we have examined a benchmark case, in which, for each sex and generation, there is a unique intensity process. To signal that, we could have used an index $x$ for the process $\lambda$, and turned it into $\lambda_{x}$. In practice, this setting could be restrictive. Indeed, we have non-OTC mortality derivatives written on a Reference population, while insurance companies face the need to hedge the longevity risk arising from their insureds, who represent the Portfolio population. In this case, basis risk arises since the two intensity processes governing the mortality of the two populations may differ. As a consequence, no perfect dynamic hedge is possible. Hence, the insurer willing to hedge her longevity risk can choose between a non-perfect dynamic hedge or a static hedge, such as a OTC longevity swap tailored to her Portfolio population, thus bearing no basis risk.

We focus on Delta-Gamma longevity risk hedging as a dynamic hedging strategy. To account for basis risk, we assume that the intensity of generation $x$ in the Reference population follows the SDE

$$
d \lambda_{x}^{n p}(t)=\left(a+b \lambda_{x}^{n p}(t)\right) d t+\sigma \sqrt{\lambda_{x}^{n p}(t)} d W_{x}(t),
$$

where, to simplify notation, we omit the subscript $x$ for the parameters. We assume that the insurance portfolio is composed by a sub-population of the Reference population. The mortality intensity of generation $x$ belonging to the Portfolio population is

$$
\lambda_{x}^{p p}(t)=\delta_{x} \lambda_{x}^{n p}(t)+\left(1-\delta_{x}\right) \lambda_{x}^{\prime}(t),
$$

with

$$
d \lambda_{x}^{\prime}(t)=\left(a^{\prime}+b^{\prime} \lambda_{x}^{\prime}(t)\right) d t+\sigma^{\prime} \sqrt{\lambda_{x}^{\prime}(t)} d W_{x}^{\prime}(t),
$$

\footnotetext{
${ }^{7}$ Here
}

$$
\begin{aligned}
\frac{\partial \ln \bar{A}(t, T)}{\partial T} & =\frac{2 \bar{a}}{\bar{\sigma}^{2}}\left[\frac{1}{2}(\bar{\gamma}+\bar{b})-\frac{\bar{\gamma} e^{\bar{\gamma}(T-t)}}{e^{\bar{\gamma}(T-t)}-1+\frac{2 \bar{\gamma}}{\bar{\gamma}+b}}\right], \\
\frac{\partial \bar{B}(t, T)}{\partial T} & =\frac{4 \bar{\gamma}^{2} e^{\bar{\gamma}(T-t)}}{\left[(\bar{\gamma}+\bar{b})\left(e^{\bar{\gamma}(T-t)}-1\right)+2 \bar{\gamma}\right]^{2}},
\end{aligned}
$$


where $W_{x}$ and $W_{x}^{\prime}$ are two independent standard Brownian Motions, $a^{\prime}>0$, $\sigma^{\prime}>0, b^{\prime} \in \mathbb{R}$ and $0 \leq \delta_{x} \leq 1$. The intensity of the insurer's Portfolio population $\lambda_{x}^{p p}$ is modelled as a convex combination of the Reference population's intensity $\lambda_{x}^{n p}$ and an idiosyncratic component $\lambda_{x}^{\prime}$ orthogonal to $\lambda_{x}^{n p}$. The idiosyncratic component $\lambda_{x}^{\prime}$ is specific to the Portfolio population and cannot be hedged by trading in the longevity bonds written on the Reference population. Intuitively, the parameter $\delta_{x}$ measures the degree of dependence between the evolution of the Portfolio population's mortality intensity and the Reference population one. Therefore, $1-\delta_{x}$ could be interpreted as a measure of basis risk. Indeed, the benchmark case without basis risk is obtained by assuming $\delta_{x}=1$. We assume $b^{\prime} \in \mathbb{R}$, thus allowing the idiosyncratic component to be either mean-reverting or not. The sign of $b^{\prime}$ will therefore be decided by the calibration that provides the lowest calibration error.

Straightforward application of Ito's Lemma shows that the dynamics of $\lambda_{x}^{p p}$ follow a two-factor CIR process. Indeed:

$$
d\left(\delta_{x} \lambda_{x}^{n p}\right)(t)=d \tilde{\lambda}_{x}^{n p}(t)=\left(\alpha+\beta \tilde{\lambda}_{x}^{n p}(t)\right) d t+\eta \sqrt{\tilde{\lambda}_{x}^{n p}(t)} d W_{x}(t),
$$

with

$$
\begin{aligned}
\alpha & =\delta_{x} a, \\
\beta & =b, \\
\eta^{2} & =\delta_{x} \sigma^{2},
\end{aligned}
$$

and

$$
d\left(\left(1-\delta_{x}\right) \lambda_{x}^{\prime}\right)(t)=d \tilde{\lambda}_{x}^{\prime}(t)=\left(\alpha^{\prime}+\beta^{\prime} \tilde{\lambda}_{x}^{\prime}(t)\right) d t+\eta^{\prime} \sqrt{\tilde{\lambda}_{x}^{\prime}(t)} d W_{x}^{\prime}(t),
$$

with

$$
\begin{aligned}
\alpha^{\prime} & =\left(1-\delta_{x}\right) a^{\prime}, \\
\beta^{\prime} & =b^{\prime}, \\
\left(\eta^{\prime}\right)^{2} & =\left(1-\delta_{x}\right)\left(\sigma^{\prime}\right)^{2} .
\end{aligned}
$$

Since $\lambda_{x}^{n p}(t)$ and $\lambda_{x}^{p p}(t)$ follow a 1 -factor and a 2-factor CIR process respectively, we can compute their conditional moments.

Assuming $0 \leq u \leq t$, in particular, the conditional covariance and correlation between $\lambda_{x}^{n p}(t)$ and $\lambda_{x}^{p p}(t)$ are

$$
\begin{aligned}
\operatorname{Cov}_{u}\left[\lambda_{x}^{p p}(t), \lambda_{x}^{n p}(t)\right] & =\delta_{x} \operatorname{Var}_{u}\left[\lambda_{x}^{n p}(t)\right], \\
\operatorname{Corr}_{u}\left[\lambda_{x}^{p p}(t), \lambda_{x}^{n p}(t)\right] & =\delta_{x} \sqrt{\frac{\operatorname{Var}_{u}\left(\lambda_{x}^{n p}(t)\right)}{\operatorname{Var}_{u}\left(\lambda_{x}^{p p}(t)\right)}},
\end{aligned}
$$


where

$$
\begin{aligned}
\operatorname{Var}_{u}\left[\lambda_{x}^{n p}(t)\right] & =\frac{a \sigma^{2}}{2 b^{2}}\left(e^{b(t-u)}-1\right)^{2}+\frac{\sigma^{2}}{b} e^{b(t-u)}\left(e^{b(t-u)}-1\right) \lambda_{x}^{n p}(u) \\
\operatorname{Var}_{u}\left[\lambda_{x}^{\prime}(t)\right] & =\frac{a^{\prime}\left(\sigma^{\prime}\right)^{2}}{2\left(b^{\prime}\right)^{2}}\left(e^{b^{\prime}(t-u)}-1\right)^{2}+\frac{\left(\sigma^{\prime}\right)^{2}}{b^{\prime}} e^{b^{\prime}(t-u)}\left(e^{b^{\prime}(t-u)}-1\right) \lambda_{x}^{\prime}(u), \\
\operatorname{Var}_{u}\left[\lambda_{x}^{p p}(t)\right] & =\delta_{x}^{2} \operatorname{Var}_{u}\left[\lambda_{x}^{n p}(t)\right]+\left(1-\delta_{x}\right)^{2} \operatorname{Var}_{u}\left[\lambda_{x}^{\prime}(t)\right]
\end{aligned}
$$

Equations (31) and (32) further clarify the interpretation of $\delta_{x}$ as a measure of the degree of comovement between the intensity of the two populations. Indeed, when $\delta_{x}=0$ the two intensities have zero correlation, while $\delta_{x}=1$ implies perfect positive correlation. $\delta_{x}$ positive ensures that $\lambda^{p p}$ is strictly positive. $\operatorname{Corr}_{u}\left[\lambda_{x}^{p p}(t), \lambda_{x}^{n p}(t)\right]$ stays between 0 and 1 . Though this may seem restrictive, this assumption is justified by the intuition that when a shock hits the Reference population, increasing for example its mortality intensity, the sub-population is affected similarly, but with a different sensitivity, while divergence between the two intensities is entirely captured by the idiosyncratic risk factor $\lambda^{\prime}$.

The survival probabilities of the Reference population are given by:

$$
S^{n p}(t, T)=A^{n p}(t, T) e^{-B^{n p}(t, T) \lambda_{x}^{n p}(t)},
$$

where $A^{n p}(t, T)$ and $B^{n p}(t, T)$ are defined as in (15). The survival probabilities of the Portfolio population can be written as functions of the common and idiosyncratic intensities as follows:

$$
\begin{aligned}
S^{p p}(t, T) & =\tilde{S}^{n p}(t, T) \tilde{S}^{\prime}(t, T) \\
& =\tilde{A}^{n p}(t, T) \tilde{A}^{\prime}(t, T) e^{-\tilde{B}^{n p}(t, T) \delta_{x} \lambda_{x}^{n p}(t)-\tilde{B}^{\prime}(t, T)\left(1-\delta_{x}\right) \lambda_{x}^{\prime}(t),}
\end{aligned}
$$

with $\tilde{A}^{n p}(t, T)=A(t, T ; \alpha, \beta, \eta), \tilde{B}^{n p}(t, T)=B(t, T ; \alpha, \beta, \eta)$ and $\tilde{A}^{\prime}(t, T)=$ $A\left(t, T ; \alpha^{\prime}, \beta^{\prime}, \eta^{\prime}\right), \tilde{B}^{\prime}(t, T)=B\left(t, T ; \alpha^{\prime}, \beta^{\prime}, \eta^{\prime}\right)$, where $\tilde{\gamma}=\sqrt{\beta^{2}+2 \eta^{2}}$ and $\tilde{\gamma}^{\prime}=\sqrt{\left(\beta^{\prime}\right)^{2}+2\left(\eta^{\prime}\right)^{2}}$.

As in the benchmark case, we use the Jarrow-Turnbull (1994) formulation of the survival probabilities in order to make their dependence on the longevity risk factor, defined as $I(t)=\lambda_{x}^{n p}-f_{x}^{n p}(0, t)$, explicit. The survival probability of the Reference population can therefore be written as:

$$
S^{n p}(t, T)=e^{-X^{n p}(t, T) I(t)+Y^{n p}(t, T)}
$$


where

$$
\begin{aligned}
X^{n p}(t, T)= & B^{n p}(t, T), \\
Y^{n p}(t, T)= & \ln A^{n p}(t, T)-B^{n p}(t, T) f_{x}^{n p}(0, t), \\
= & \ln A^{n p}(t, T)-B^{n p}(t, T)\left[-\left.\frac{\partial \ln A^{n p}(t, T)}{\partial T}\right|_{(0, t)}+\right. \\
& \left.+\left.\lambda_{x}^{n p}(0) \frac{\partial B^{n p}(t, T)}{\partial t}\right|_{(0, t)}\right],
\end{aligned}
$$

and

$$
\begin{aligned}
\frac{\partial \ln A^{n p}(t, T)}{\partial T} & =\frac{2 a}{\sigma^{2}}\left[\frac{1}{2}(\gamma-b)-\frac{\gamma e^{\gamma(T-t)}}{e^{\gamma(T-t)}-1+\frac{2 \gamma}{\gamma-b}}\right] \\
\frac{\partial B^{n p}(t, T)}{\partial T} & =\frac{4 \gamma^{2} e^{\gamma(T-t)}}{\left[(\gamma-b)\left(e^{\gamma(T-t)}-1\right)+2 \gamma\right]^{2}} .
\end{aligned}
$$

Similarly, for the survival probabilities of the Portfolio population we have:

$$
S^{p p}(t, T)=e^{-X^{p p}(t, T) \delta_{x} I(t)-X^{\prime}(t, T)\left(1-\delta_{x}\right) \lambda_{x}^{\prime}(t)+Y^{p p}(t, T)},
$$

where

$$
\begin{aligned}
X^{p p}(t, T) & =\tilde{B}^{n p}(t, T), \\
X^{\prime}(t, T) & =\tilde{B}^{\prime}(t, T), \\
Y^{p p}(t, T) & =\ln \tilde{A}^{n p}(t, T)+\ln \tilde{A}^{\prime}(t, T)-\tilde{B}^{n p}(t, T) f_{x}^{n p}(0, t) .
\end{aligned}
$$

\section{Sensitivity of the insurance portfolio}

We assume that the liabilities of the insurance company are represented by an annuity contract ${ }^{8}$ - with maturity $T$ and annual installments $R$ paid at year-end - written on an individual belonging to the Portfolio population, aged $x$ at time 0 . The extension to term insurance, pure endowments or other non-indexed contracts is straightforward.

For the case without basis risk, the fair value of the annuity contract, which is also the value of its reserve, depends at any time $0 \leq t \leq T$ on the mortality intensity $\lambda_{x}$ in equation (1). Thus, using the Jarrow and Turnbull (1994) representation, we can write:

\footnotetext{
${ }^{8}$ Indeed, for simplicity, we abstract from idiosyncratic risk and consider the annuity as a perfectly-diversified portfolio on annuities issued on homogeneous individuals.
} 


$$
\begin{aligned}
N(t, T) & =R \sum_{u=1}^{T-t} D(t, t+u) S(t, t+u), \\
& =R \sum_{u=1}^{T-t} e^{-\bar{X}(t, t+u) J(t)+\bar{Y}(t, t+u)} \cdot e^{-X(t, t+u) I(t)+Y(t, t+u)} .
\end{aligned}
$$

The marginal effect on the value of the reserve caused by any unexpected change in the common mortality risk factor or in the interest rate process, can approximated as follows:

$$
d N=\frac{\partial N}{\partial t} d t+\frac{\partial N}{\partial I} d I+\frac{1}{2} \frac{\partial^{2} N}{\partial I^{2}}(d I)^{2}+\frac{\partial N}{\partial J} d J+\frac{1}{2} \frac{\partial^{2} N}{\partial J^{2}}(d J)^{2},
$$

where

$$
\begin{gathered}
\frac{\partial N}{\partial I}=R \sum_{u=1}^{T-t} D(t, t+u) \Delta^{M}(t, t+u), \\
\frac{\partial^{2} N}{\partial I^{2}}=R \sum_{u=1}^{T-t} D(t, t+u) \Gamma^{M}(t, t+u), \\
\frac{\partial N}{\partial J}=R \sum_{u=1}^{T-t} \Delta^{F}(t, t+u) S(t, t+u), \\
\frac{\partial^{2} N}{\partial J^{2}}=R \sum_{u=1}^{T-t} \Gamma^{F}(t, t+u) S(t, t+u),
\end{gathered}
$$

with

$$
\begin{aligned}
\Delta^{M}(t, T) & :=\frac{\partial S(t, T)}{\partial I}=-X(t, T) S(t, T) \leq 0, \\
\Gamma^{M}(t, T) & :=\frac{\partial^{2} S(t, T)}{\partial I^{2}}=X(t, T)^{2} S(t, T) \geq 0, \\
\Delta^{F}(t, T) & :=\frac{\partial D(t, T)}{\partial J}=-\bar{X}(t, T) D(t, T) \leq 0, \\
\Gamma^{F}(t, T) & :=\frac{\partial^{2} D(t, T)}{\partial J^{2}}=\bar{X}(t, T)^{2} D(t, T) \geq 0 .
\end{aligned}
$$

The signs of these Greeks help understand the effects of a mortality or a financial shock on the price of the annuity. The negative sign of $\Delta^{M}$ and $\Delta^{F}$ shows that, as one would expect, the value of the annuity is decreasing in both the longevity and the interest rate risk factors. The positivity of $\Gamma^{M}$ and $\Gamma^{F}$ indicates, instead, that the higher $I$ or $J$, the higher is the annuity value sensitivity to their changes. 
If instead there is basis risk, then at any time $0 \leq t \leq T$, the fair value of the annuity contract is driven by the mortality intensity $\lambda_{x}^{p p}$ and can be written as:

$$
N^{p p}(t, T)=R \sum_{u=1}^{T-t} D(t, t+u) S^{p p}(t, t+u),
$$

or equivalently as:

$$
\begin{aligned}
& N^{p p}(t, T)= \\
& =R \sum_{u=1}^{T-t} e^{-\bar{X}(t, t+u) J(t)+\bar{Y}(t, t+u)} \cdot e^{-X^{p p}(t, t+u) \delta_{x} I(t)-X^{\prime}(t, t+u)\left(1-\delta_{x}\right) \lambda_{x}^{\prime}(t)+Y^{p p}(t, t+u)} .
\end{aligned}
$$

Under the previous assumptions, if there is any unexpected change in the common mortality risk factor, in the idiosyncratic component or in the interest rate process, the marginal effect on the reserve is as follows:

$$
\begin{aligned}
d N^{p p}=\frac{\partial N^{p p}}{\partial t} d t+\frac{\partial N^{p p}}{\partial I} d I & +\frac{1}{2} \frac{\partial^{2} N^{p p}}{\partial I^{2}}(d I)^{2}+\frac{\partial N^{p p}}{\partial \lambda^{\prime}} d \lambda^{\prime}+\frac{1}{2} \frac{\partial^{2} N^{p p}}{\partial\left(\lambda^{\prime}\right)^{2}}\left(d \lambda^{\prime}\right)^{2}+ \\
& +\frac{\partial N^{p p}}{\partial J} d J+\frac{1}{2} \frac{\partial^{2} N^{p p}}{\partial J^{2}}(d J)^{2}
\end{aligned}
$$

where

$$
\begin{aligned}
& \frac{\partial N^{p p}}{\partial I}=R \sum_{u=1}^{T-t} D(t, t+u) \Delta_{p p}^{M}(t, t+u), \\
& \frac{\partial^{2} N^{p p}}{\partial I^{2}}=R \sum_{u=1}^{T-t} D(t, t+u) \Gamma_{p p}^{M}(t, t+u), \\
& \frac{\partial N^{p p}}{\partial \lambda^{\prime}}=R \sum_{u=1}^{T-t} D(t, t+u) \Delta_{p p}^{\prime}(t, t+u), \\
& \frac{\partial^{2} N^{p p}}{\partial\left(\lambda^{\prime}\right)^{2}}=R \sum_{u=1}^{T-t} D(t, t+u) \Gamma_{p p}^{\prime}(t, t+u), \\
& \frac{\partial N^{p p}}{\partial J}=R \sum_{u=1}^{T-t} \Delta^{F}(t, t+u) S^{p p}(t, t+u), \\
& \frac{\partial^{2} N^{p p}}{\partial J^{2}}=R \sum_{u=1}^{T-t} \Gamma^{F}(t, t+u) S^{p p}(t, t+u),
\end{aligned}
$$


with

$$
\begin{aligned}
\Delta_{p p}^{M}(t, T) & :=\frac{\partial S^{p p}(t, T)}{\partial I}=-X^{p p}(t, T) \delta_{x} S^{p p}(t, T) \leq 0 \\
\Gamma_{p p}^{M}(t, T) & :=\frac{\partial^{2} S^{p p}(t, T)}{\partial I^{2}}=\left(X^{p p}(t, T) \delta_{x}\right)^{2} S^{p p}(t, T) \geq 0 \\
\Delta_{p p}^{\prime}(t, T) & :=\frac{\partial S^{p p}(t, T)}{\partial \lambda^{\prime}}=-X^{\prime}(t, T)\left(1-\delta_{x}\right) S^{p p}(t, T) \leq 0 \\
\Gamma_{p p}^{\prime}(t, T) & :=\frac{\partial^{2} S^{p p}(t, T)}{\partial\left(\lambda^{\prime}\right)^{2}}=\left(X^{\prime}(t, T)\left(1-\delta_{x}\right)\right)^{2} S^{p p}(t, T) \geq 0 \\
\Delta^{F}(t, T) & :=\frac{\partial D(t, T)}{\partial J}=-\bar{X}(t, T) D(t, T) \leq 0 \\
\Gamma^{F}(t, T) & :=\frac{\partial^{2} D(t, T)}{\partial J^{2}}=\bar{X}(t, T)^{2} D(t, T) \geq 0
\end{aligned}
$$

The same comments about the signs of the Delta and Gamma of the annuity for the case with no basis risk, apply here. Moreover, from equation (58), we can also observe that the sensitivity of the annuity with respect to the common longevity risk factor $I$ is directly proportional to the parameter $\delta_{x}$.

\section{$6 \quad$ Static Hedging Strategies}

In order to hedge the unexpected changes formalized above, the insurance company can buy a static, OTC hedge, provided by a so-called $s$-swap or longevity swap, usually customized, or written on the Portfolio population. A longevity swap is a sequence of $s$-forwards. An $s$-forward signed at $t$ with maturity $T$ is a contract in which - for reasons to be explained below one party agrees to pay a fixed amount $K(T)$ in exchange for the number of survivors at $\mathrm{T}$ belonging to a specific generation $x$ of the Portfolio population. We normalize the number of individuals in generation $x$ to one. We thus abstract from annuitant-specific risk and consider a single annuity as equivalent to a well-diversified homogeneous portfolio of annuities. If the maturity of the forward is $T$, and the fixed payment is $K(T)$, then the payoff at maturity, from the point of view of who pays fixed, is

$$
\exp \left(-\int_{t}^{T} \lambda_{x}^{p p}(s) d s\right)-K(T)
$$

An $s$-forward (unit hedge) helps providers of annuities to hedge their exposure: if the provider has sold a pure endowment on generation $x$ with maturity $T$, and buys an $s$-forward, he will pay $K(T)$ for sure instead of being exposed to the randomness of the payment, $\exp \left(-\int_{t}^{T} \lambda_{x}^{p p}(s) d s\right)$. Under the assumption of no arbitrage, and assuming independence between 
mortality and interest-rate risk, the fair value at time $t$ of such a contract is

$$
\begin{aligned}
& {[S(t, T)-K(T)] D(t, T)=} \\
= & \mathbb{E}_{t}\left[\exp \left(-\int_{t}^{T} \lambda_{x}^{p p}(s) d s\right)-K(T)\right] \mathbb{E}_{t}\left[\exp \left(-\int_{t}^{T} r(u) d u\right)\right] .
\end{aligned}
$$

where index $t$ signals that the expectation is the $\mathcal{F}_{t}-$ one. Since, in order to enter such a contract, no price is paid at inception, the no-arbitrage value of $K(T)$, which equates the fair value to zero, is $S(t, T)$.

A longevity swap is a sequence of $s$-forwards. If the exchange of amounts happens once a year, the payment for the period $(T-1, T)$ is $K(T)$ and the contract lasts until the last individual of the generation is dead (at age $\omega$ ), the payoffs are given by (64) for $T=1, . ., \omega-t$. Under the assumption of no arbitrage, and still assuming independence between mortality and interestrate risk, the value at time $t$ of such a contract is

$$
\begin{aligned}
\sum_{T=t+1}^{\omega-t}[S(t, T)-K(T)] D(t, T)= \\
\quad=\sum_{T=t+1}^{\omega-t} \mathbb{E}_{t}\left[\exp \left(-\int_{t}^{T} \lambda_{x}^{p p}(s) d s\right)-K(T)\right] \mathbb{E}_{t}\left[\exp \left(-\int_{t}^{T} r(u) d u\right)\right],
\end{aligned}
$$

which is equal to zero, as a fair pricing would require, if $K(T)$ is set equal to the survival probability up to time $T$. We call $K(T)$ the swap rate for the time period $(T-1, T) .9$

Usually, the previous swap is not offered to the insurance company at fair value. It entails a cost, which we take to be fixed and equal to $C_{0}$. It follows that the fees $K(T)$ are raised to $K^{\prime}(T)$, where the sequence $K^{\prime}(T)$ solves

$$
-C_{0}=\sum_{T=t+1}^{\omega-t}\left[S(t, T)-K^{\prime}(T)\right] D(t, T) .
$$

For the sake of simplicity, we assume that the cost $C_{0}$ is evenly distributed along the "life" of the swap, by increasing the swap rates $K$ by the same amount, i.e. $K^{\prime}(T)=K(T)(1+m)=S(t, T)(1+m)$ where the premium

\footnotetext{
${ }^{9}$ An alternative would be to fix a unique swap rate for all periods, $K(T)=K$. In this case fairness would be guaranteed by setting $K$ equal to the following value:

$$
K=\frac{\sum_{T=t+1}^{\omega-t} \mathbb{E}\left[\exp \left(-\int_{t}^{T} \lambda_{x}(s) d s\right)\right] \mathbb{E}\left[-\exp \left(\int_{t}^{T} r(u) d u\right)\right]}{\sum_{T=1}^{\omega-t} \mathbb{E}\left[-\exp \left(\int_{t}^{T} r(u) d u\right)\right]} .
$$
}


loading $m$ is determined as follows:

$$
\begin{aligned}
m & =\frac{C_{0}}{\sum_{T=t+1}^{\omega-t} S(t, T) D(t, T)} \\
& =\frac{C_{0}}{\sum_{T=t+1}^{\omega-t} e^{-\bar{X}(t, T) J(t)+\bar{Y}(t, T)} \cdot e^{-X(t, T) I(t)+Y(t, T)}} .
\end{aligned}
$$

In principle, the insurance company can be interested in hedging interest rate risk too. We neglect this coverage here. However, given the similarity of the two processes, the formulas for an interest rate swap would be similar to the survival one.

\section{Greeks and Delta-Gamma hedging strategy}

An alternative to the previous hedge is a dynamic hedging strategy that uses longevity bonds based on the survivorship of a Reference population 10 .

We consider two different longevity risk hedging strategies: the DeltaGamma strategy (see Luciano et al. (2012)), and an extension called DeltaGamma-Theta hedging strategy. The first one covers both the first and second-order changes in the reserve, Delta and Gamma, that depend on the changes of the CIR longevity intensity, using a portfolio composed of longevity bonds. The second one adds a risk-free zero-coupon bond to the hedging portfolio in order to cover also the time-derivative (the Theta) of the value of the reserve. For consistency with the static hedge, we assume that interest rate risk is not covered. For the same reason, we also assume that the dynamic hedge is self-financing, a requirement formalized below. At each rebalancing date we re-apply the self-financing Delta-Gamma(-Theta) strategy using the same instruments. Immediately before each rebalancing date $t$ we evaluate the portfolio. Its value is the gain or loss of the hedging strategy, which we finance through the bank account. In other words, any gain or loss from the hedging revision is stored or charged in the bank account, from which the payments due because of the annuity contract are also taken. The bank account accrues or charges the short interest rate $r(t)$. As customary in this literature, we refer to the absolute value of the bank account as to the hedging error of the dynamic strategy.

\footnotetext{
${ }^{10}$ We can use a number of other instruments to cover the annuity, starting from life assurances or death bonds, which pay the benefit in case of death. We restrict the attention to longevity bonds for the sake of simplicity, whose payoff for the maturity $T$ is

$$
\exp \left(-\int_{t}^{T} \lambda_{x}^{n p}(s) d s\right) .
$$
}




\subsection{No basis risk}

Assume first that there is no basis risk, so that $\delta_{x}=1$. Under no-arbitrage, if the longevity bond maturity is $T_{i}$, its fair value at time $t, M_{i}(t)$, is

$$
M_{i}(t)=S\left(t, T_{i}\right) D\left(t, T_{i}\right),
$$

which, using the CIR assumption, can be written as

$$
M_{i}(t)=\bar{A}\left(t, T_{i}\right) e^{-\bar{B}\left(t, T_{i}\right) r(t)} A\left(t, T_{i}\right) e^{-B\left(t, T_{i}\right) \lambda_{x}(t)},
$$

or, in the Jarrow and Turnbull formulation, as

$$
M_{i}(t)=e^{-\bar{X}\left(t, T_{i}\right) J(t)+\bar{Y}\left(t, T_{i}\right)} e^{-X\left(t, T_{i}\right) I(t)+Y\left(t, T_{i}\right)} .
$$

The dynamics of $M_{i}(t)$ are driven by $\lambda_{x}(t)$ and $r(t)$, and can be written as follows:

$$
d M_{i}=\frac{\partial M_{i}}{\partial t} d t+\frac{\partial M_{i}}{\partial I} d I+\frac{1}{2} \frac{\partial^{2} M_{i}}{\partial I^{2}}(d I)^{2}+\frac{\partial M_{i}}{\partial J} d J+\frac{1}{2} \frac{\partial^{2} M_{i}}{\partial J^{2}}(d J)^{2},
$$

where

$$
\begin{aligned}
\frac{\partial M_{i}}{\partial I} & =D\left(t, T_{i}\right) \Delta^{M}\left(t, T_{i}\right), \\
\frac{\partial^{2} M_{i}}{\partial I^{2}} & =D\left(t, T_{i}\right) \Gamma^{M}\left(t, T_{i}\right), \\
\frac{\partial M_{i}}{\partial J} & =\Delta^{F}\left(t, T_{i}\right) S\left(t, T_{i}\right), \\
\frac{\partial^{2} M_{i}}{\partial J^{2}} & =\Gamma^{F}\left(t, T_{i}\right) S\left(t, T_{i}\right),
\end{aligned}
$$

where $\Delta^{M}(t, T), \Gamma^{M}(t, T), \Delta^{F}(t, T), \Gamma^{F}(t, T)$ are given by 50), 51, 52, 53, respectively.

The dynamics of the annuity $N(t)$ is driven by the same factors:

$$
d N=\frac{\partial N}{\partial t} d t+\frac{\partial N}{\partial I} d I+\frac{1}{2} \frac{\partial^{2} N}{\partial I^{2}}(d I)^{2}+\frac{\partial N}{\partial J} d J+\frac{1}{2} \frac{\partial^{2} N}{\partial J^{2}}(d J)^{2} .
$$

In order to Delta-Gamma hedge and keep the hedge self financing, we need three bonds at each point in time. Their maturities $T_{i}, i=1,2,3$ are kept constant along the life of the hedge. The number of bonds in the portfolio at $t$ is $n_{i}, i=1,2,3$.

At each rebalancing point $t$, the positions in the bonds used to hedge solve the following system

$$
\left\{\begin{array}{rlr}
-\frac{\partial N(t)}{\partial I} d I+\sum_{i=1}^{3} n_{i}(t) \frac{\partial M_{i}(t)}{\partial I} d I & =0 \\
-\frac{\partial^{2} N(t)}{\partial I^{2}}(d I)^{2}+\sum_{i=1}^{3} n_{i}(t) \frac{\partial^{2} M_{i}(t)}{\partial I^{2}}(d I)^{2} & =0 \\
-\frac{\partial N(t)}{\partial t} d t & +\sum_{i=1}^{3} n_{i}(t) \frac{\partial M_{i}(t)}{\partial t} d t & +n_{4} \frac{\partial Z(t)}{\partial t}=0 \\
-N(t)+\sum_{i=1}^{3} n_{i}(t) M_{i}(t) & +n_{4} Z(t)=0
\end{array}\right.
$$


The first equation nullifies the Delta of the portfolio, the second nullifies the Gamma, while the third is a self-financing condition. The terms associated to the annuity enter with negative signs, as they represent the liability that the company is endowed with. The longevity bond value is equal to a pure endowment. The difference, from the standpoint of an insurance company, is that it can sell annuities and pure endowments - or reduce its exposure through reinsurance - and buy longevity bonds, while, at least in principle, it cannot do the converse 11

An extension of the previous strategy, that we call Delta-Gamma-Theta strategy, aims at covering also the deterministic changes $\frac{\partial N}{\partial t}$ in the value of the annuity. The strategy requires an additional asset in the hedging portfolio. We consider a risk-less zero-coupon bond $Z(t)$, whose maturity coincides with the rebalancing frequency of the dynamic strategy. Then, at each evaluation date $t$, the hedging portfolio solves the following system of 4 equations in 4 unknowns:

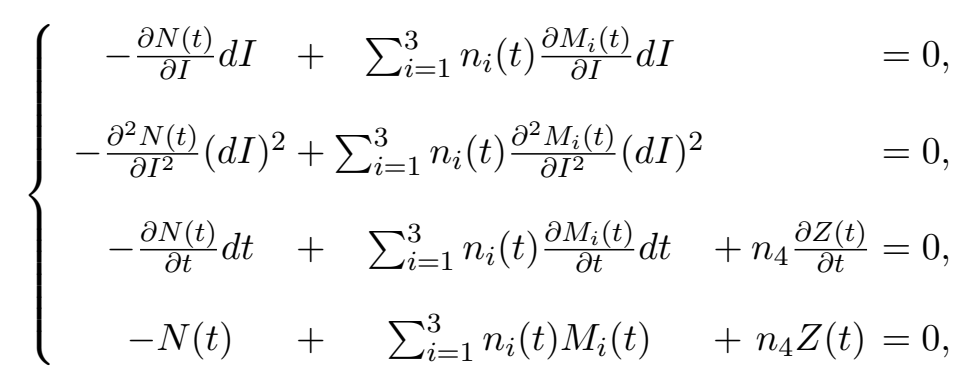

where the third equation of system (67) nullifies the time-sensitivity of the portfolio. The zero-coupon bond $Z(t)$ does not appear in the first two equations because it does not depend on the longevity risk factor $I(t)$ and hence its Delta and Gamma are zero.

\subsection{Basis risk}

When there is basis risk $\left(\delta_{x}<1\right)$, the dynamics of the annuity contract $N^{p p}(t)$ written on the Portfolio population are given by equation (57). We assume that at least three longevity bonds, with different maturities $T_{1}, T_{2}, T_{3}$, written on generation $x$ of the Reference population are traded. For each $i=1,2,3$, the value of the longevity bond, $M_{i}^{n p}\left(t, T_{i}\right)$ at any time $0 \leq t \leq T_{i}$, depends on $\lambda_{x}^{n p}$ and is given by:

$$
\begin{aligned}
M_{i}^{n p}(t) & =D\left(t, T_{i}\right) S^{n p}\left(t, T_{i}\right), \\
& =e^{-\bar{X}\left(t, T_{i}\right) J(t)+\bar{Y}\left(t, T_{i}\right)} \cdot e^{-X^{n p}\left(t, T_{i}\right) I(t)+Y^{n p}\left(t, T_{i}\right)}
\end{aligned}
$$

\footnotetext{
${ }^{11}$ Reinsurance companies have less constraints in this respect. For instance, they can swap pure endowments or issue longevity bonds: see for instance Cowley and Cummins (2005).
} 
while its dynamics can be written as

$$
d M_{i}^{n p}=\frac{\partial M_{i}^{n p}}{\partial t} d t+\frac{\partial M_{i}^{n p}}{\partial I} d I+\frac{1}{2} \frac{\partial^{2} M_{i}^{n p}}{\partial I^{2}}(d I)^{2}+\frac{\partial M_{i}^{n p}}{\partial J} d J+\frac{1}{2} \frac{\partial^{2} M_{i}^{n p}}{\partial J^{2}}(d J)^{2} .
$$

A perfect hedge of longevity risk cannot be achieved, even with continuoustime trading because changes in $\lambda^{\prime}$ affect $N^{p p}$, but not $M^{n p}$ as the comparison between (57) and (69) shows. The fact that we cannot hedge changes in the idiosyncratic risk factor $\lambda_{x}^{\prime}(t)$ means that, in general, the value of the hedging portfolio will not perfectly replicate the value of the insurance liabilities, i.e. the overall hedging error will differ from zero. A nice feature of our model is the ability to separate the source of risk that can still be hedged, i.e. $I(t)$, from the source of risk that remains unhedgeable, i.e. $\lambda_{x}^{\prime}(t)$. This property allows us to use the longevity bonds $M_{i}^{n p}\left(t, T_{i}\right)$ as hedging instruments and to perform either a Delta-Gamma or a Delta-Gamma-Theta hedging strategy in order to hedge unexpected changes in the common longevity risk factor $I(t)$.

In this case, the Delta-Gamma hedging portfolio is determined by solving the following system

$$
\left\{\begin{aligned}
-\frac{\partial N^{p p}(t)}{\partial I} d I+\sum_{i=1}^{3} n_{i} \frac{\partial M_{i}^{n p}(t)}{\partial I} d I & =0 \\
-\frac{\partial^{2} N^{p p}(t)}{\partial I^{2}}(d I)^{2}+\sum_{i=1}^{3} n_{i} \frac{\partial^{2} M_{i}^{n p}(t)}{\partial I^{2}}(d I)^{2} & =0 \\
-N^{p p}(t)+\sum_{i=1}^{3} n_{i} M_{i}^{n p}(t) & =0
\end{aligned}\right.
$$

while the Delta-Gamma-Theta hedging portfolio solves

$$
\left\{\begin{array}{rlr}
-\frac{\partial N^{p p}(t)}{\partial I} d I+\sum_{i=1}^{3} n_{i} \frac{\partial M_{i}^{n p}(t)}{\partial I} d I & =0 \\
-\frac{\partial^{2} N^{p p}(t)}{\partial I^{2}}(d I)^{2}+\sum_{i=1}^{3} n_{i} \frac{\partial^{2} M_{i}^{n p}(t)}{\partial I^{2}}(d I)^{2} & =0 \\
-\frac{\partial N^{p p}(t)}{\partial t} d t & +\sum_{i=1}^{3} n_{i} \frac{\partial M_{i}^{n p}(t)}{\partial t} d t & +n_{4} \frac{\partial Z(t)}{\partial t}=0 \\
-N^{p p}(t)+\sum_{i=1}^{3} n_{i} M_{i}^{n p}(t) & +n_{4} Z(t)=0
\end{array}\right.
$$

\section{Hedging Strategies: effectiveness and performance comparison}

In order to compare the static and dynamic hedges described above we proceed as follows: we calibrate the models to the observed mortality rates of 65-year old UK males, we determine the cost of the static hedge which would equate a given percentile of the hedging error of a dynamic strategy, under different assumptions on its rebalancing frequency, in the presence and 


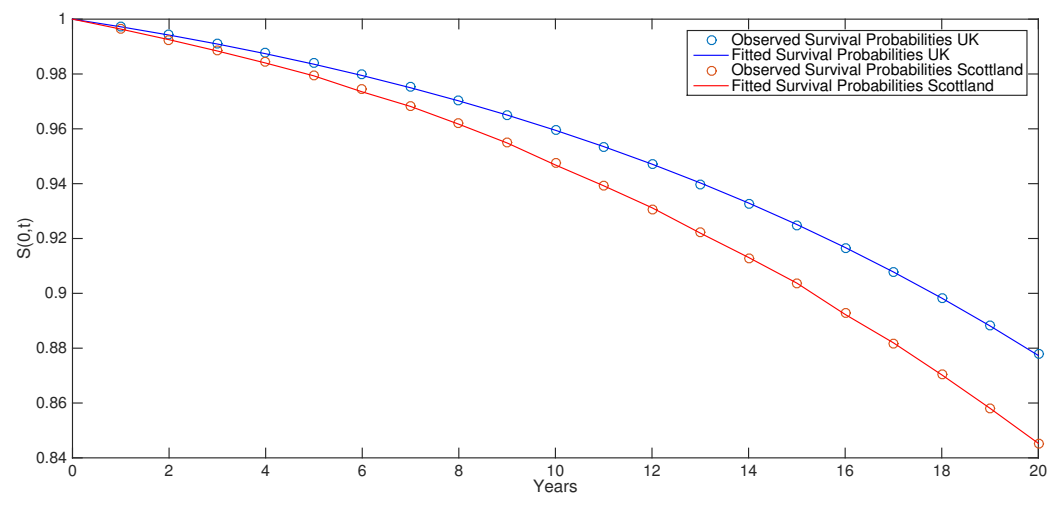

Figure 1. Observed and fitted survival probabilities for the Reference and the Portfolio Population.

in the absence of basis risk. We call this a value-at-risk loading principle. Consistently with the above, we implicitly assume that interest-rate risk has already been hedged perfectly or does not exist.

\subsection{Calibration}

We calibrate the parameters of our Reference (Portfolio) mortality model to the generation of UK (Scottish) males born in 1946, who were aged 64 on $31 / 12 / 2010$ (i.e. $x=65$ ). Under the constraint given by condition (2), we fix $01 / 01 / 1991$ as the observation point (individuals have all reached aged $44)$ and we fit the observed survival probabilities $S^{n p}(0, t), S^{p p}(0, t)$ with $\mathrm{t}=1, \ldots 20$. We fit our models minimizing the Rooted Mean Squared Error (RMSE) between the model-implied and the observed survival probabilities.

We perform two different calibrations. The first calibration fits only the parameters of the Reference Population, using the Human Mortality Database data for UK. The resulting parameters, which are collected - together with the calibration error - in Table 1, are used to simulate the dynamic Delta-Gamma-Theta hedging strategy without basis risk.

Table 1. Reference Population calibration results.

\begin{tabular}{cccc}
\hline$a$ & $b$ & $\sigma$ & Calibration Error \\
\hline $4.13 \cdot 10^{-5}$ & 0.0709 & 0.0087 & 0.00006 \\
\hline
\end{tabular}

Because condition (2) holds, the simulated mortality intensities $\lambda_{x}^{n p}(t)$ will be strictly positive. In the simulations, we assume that the maximum life-span of an individual belonging to generation $x$ is $\omega=115$, hence the time horizon we use for the simulations of the intensity process is 50 years. The 

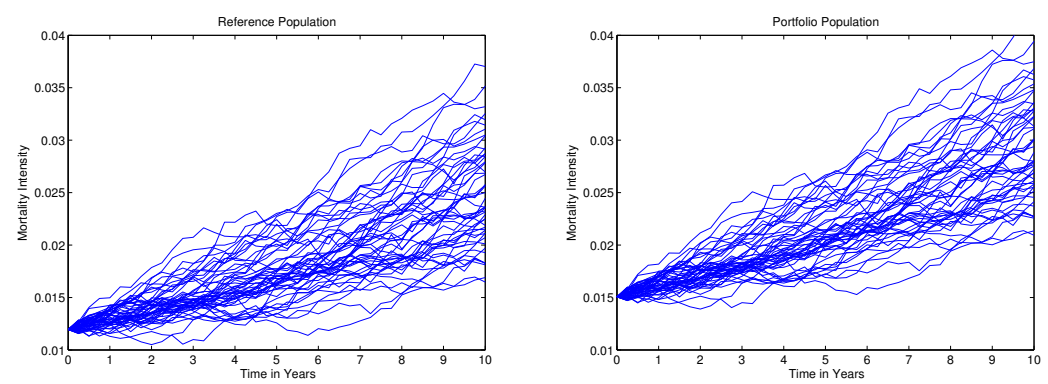

Figure 2. On the left-hand side, sample paths of the Reference population intensity process $\lambda_{x}^{n p}(t)$. On the right-hand side, sample paths of the Portfolio population intensity process $\lambda_{x}^{p p}(t)$.

second calibration fits the parameters of the Reference and of the Portfolio populations jointly, and is used for the simulations of the dynamic hedging with basis risk. As for the Portfolio population, we use Human Mortality Database data for 65-year-old Scottish males. This population is included in the more general UK dataset and therefore is suitable to act as a subpopulation in our example. The calibration error is 0.00015 , and the values of the calibrated parameters are shown in Table 2

Figure 1 shows the observed and the fitted survival probabilities for the Reference and the Portfolio populations. Simulated sample paths of both the $\lambda_{x}^{n p}(t)$ and $\lambda_{x}^{p p}(t)$ processes are shown in Figure 2.

Table 2. Reference and Portfolio population joint calibration results.

\begin{tabular}{ccccccc}
\hline$a$ & $b$ & $\sigma$ & $\delta_{x}$ & $a^{\prime}$ & $b^{\prime}$ & $\sigma^{\prime}$ \\
\hline $3.3357 \cdot 10^{-5}$ & 0.0727 & 0.0082 & 0.9897 & 0.0077 & 0.0155 & $4.4463 \cdot 10^{-08}$ \\
\hline
\end{tabular}

We set the interest rate to a constant value $r=0.02$.

\subsection{Rebalancing frequency and dynamic hedging performance without basis risk}

In this section we compute the performance of the dynamic hedging strategy we described in Section 7 under different rebalancing frequencies. We use the results to assess reasonable ranges for the cost of a longevity swap, as described in Section 6, when basis risk is negligible. Let us consider an annuity provider who has sold a whole-life annuity written on UK males aged 65 at time 0 . Three longevity bonds with rolling maturities 10, 15 and 20 years, written on the same generation of 65-year-old UK males, and a risk-free zero-coupon bond with rolling maturity equal to the rebalancing frequency of the dynamic hedge exist.

We evaluate the effectiveness of the self-financing dynamic Delta-GammaTheta hedging strategy fixing the time horizon to 30 years. We consider 
three different rebalancing frequencies of 3 months, 6 months and 1 year, respectively.

Figure 3 shows the simulated percentiles, from the 5 th to the 95 th, of the distribution of the bank account over time. The value of the bank account is determined, at each rebalancing date, by crediting (debiting) any gain (loss) due to the difference between the hedging portfolio value before rebalancing and the annuity value.

Figure 4 reports the distribution of the bank account after 30 years, for the three different rebalancing frequencies. The picture shows that - as expected - the average cost of the hedging strategy is higher the longer the time interval between two revisions of the strategy. Also, increasing the rebalancing frequency reduces remarkably the dispersion of the value around its mean. The strategy rebalanced at 1-year frequency presents the fattest tails. Table 3 contains, for each case, the mean and standard deviation of the hedging error after 30 years and allows to appreciate the effects of different rebalancing frequencies 12

Given the results of our implementation of the dynamic hedging strategy, we compute the cost of the swap based on a value-at-risk loading principle. More precisely, the premium charged to the buyer of the swap $C_{0}$ is computed as the present value of the $99.5 \%$ value-at-risk of the bank account value at $t=30$ years obtained applying our hedging strategy with different rebalancing intervals. Table 5 reports the $\operatorname{cost} C_{0}$ and loading $m$ of the swap. The loading $m$, which represents the percentage increase in each observed survival, ranges from $0.01 \%$ to $0.06 \%$. This value might seem low, but it is a benchmark value, since it is obtained in the absence of transaction costs and basis risk.

\begin{tabular}{|c|c|c|c|c|c|c|c|}
\hline & 3 months & 6 months & 1 year & & 3 months & 6 months & 1 year \\
\hline Mean & 0.00073 & 0.00142 & 0.00274 & Mean & 0.00073 & 0.00142 & 0.00275 \\
\hline Std & 0.00068 & 0.00140 & 0.00300 & Std & 0.00067 & 0.00143 & 0.00301 \\
\hline
\end{tabular}

Table 3. Moments of the hedging error of the Delta-Gamma-Theta strategy under different rebalancing frequencies.

Table 4. Moments of the hedging error of the Delta-Gamma strategy under different rebalancing frequencies.

Tables 4 and 6 provide the mean, standard deviations, premiums and loadings for the case in which Theta hedging is not performed. They show that there is not much difference in terms of results between performing a Delta-Gamma or a Delta-Gamma-Theta hedge. Both strategies provide similar hedging errors, but the Delta-Gamma-Theta requires an additional asset which could increase the overall cost of the strategy if transaction costs were taken into account.

\footnotetext{
${ }^{12}$ We remark that this result is obtained in the absence of transaction costs, which we neglect here and will be higher the higher the frequency.
} 

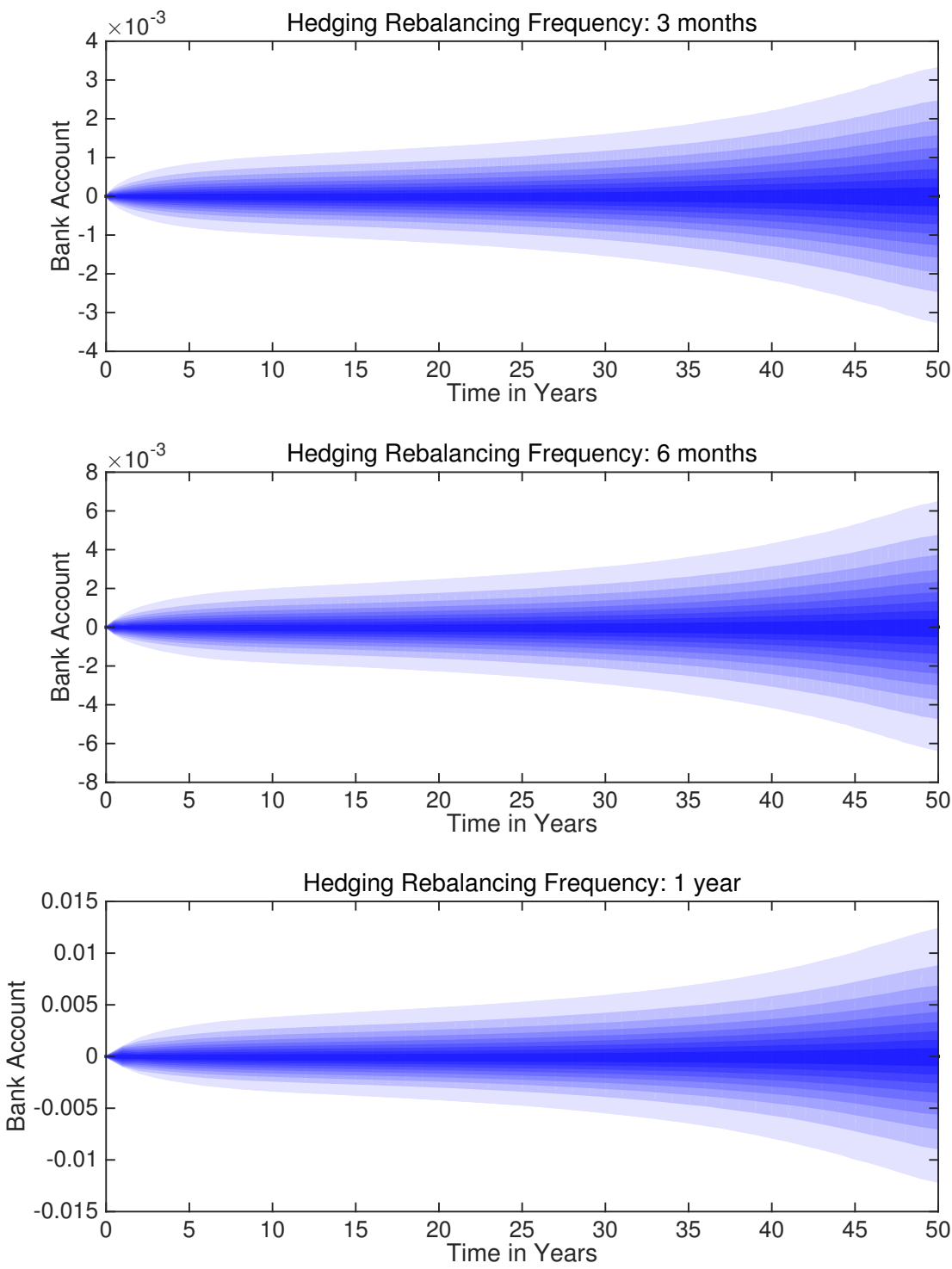

Figure 3. Percentiles of the bank account under different assumptions on the hedging rebalancing frequency. 


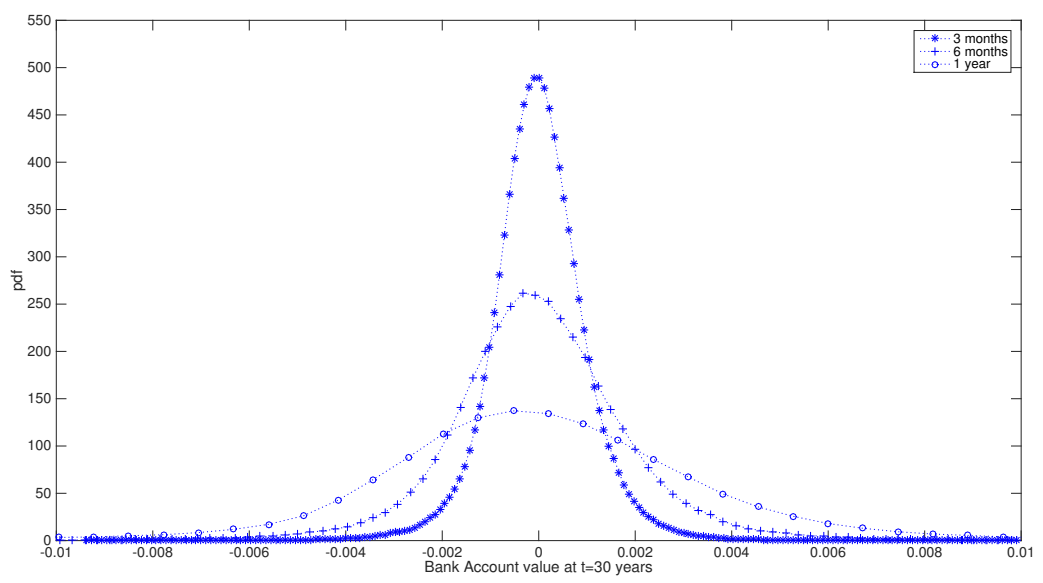

Figure 4. Distribution of the value of the bank account at $t=30$ years for different rebalancing frequencies.

\begin{tabular}{cccc}
\hline & 3 months & 6 months & 1 year \\
\hline$C_{0}$ & 0.00209 & 0.00447 & 0.01027 \\
$m$ & $0.01 \%$ & $0.02 \%$ & $0.06 \%$ \\
\hline
\end{tabular}

Table 5. Longevity Swap premiums and loadings equivalent to the $99.5 \%$ Value-atRisk of the Delta-Gamma-Theta Hedging strategy at $t=30$ years.

\begin{tabular}{cccc}
\hline & 3 months & 6 months & 1 year \\
\hline$C_{0}$ & 0.00207 & 0.00470 & 0.01012 \\
$m$ & $0.01 \%$ & $0.03 \%$ & $0.06 \%$ \\
\hline
\end{tabular}

Table 6. Longevity Swap premiums and loadings equivalent to the $99.5 \%$ Value-atRisk of the Delta-Gamma Hedging strategy at $t=30$ years.

\subsection{Rebalancing frequency and dynamic hedging performance with basis risk}

In this section we examine the performance of a dynamic Delta-GammaTheta hedging strategy when basis risk is present. We assume that an annuity provider has sold a whole-life annuity written on Scottish males aged 65 at time 0 . As in the previous section, together with a risk-free zero-coupon bond, we assume that three longevity bonds written on the generation of 65 year-old UK males are traded in the market. The two populations follow now different processes, as described in Section 4 Basis risk enters the picture, and a dynamic perfect hedge is not possible.

The hedging error is caused both by the presence of basis risk and by the discrete-time rebalancing of the dynamic hedging strategy. It can be seen from Figure 5 that the bank account is not perfectly centered at zero and that its absolute value is higher than in the case when no basis risk is present, because the idiosyncratic component cannot be hedged. As in the previous case, for each rebalancing frequency, the plot is a fan chart representing the percentiles (from the 5th to the 95th) of the distribution of 

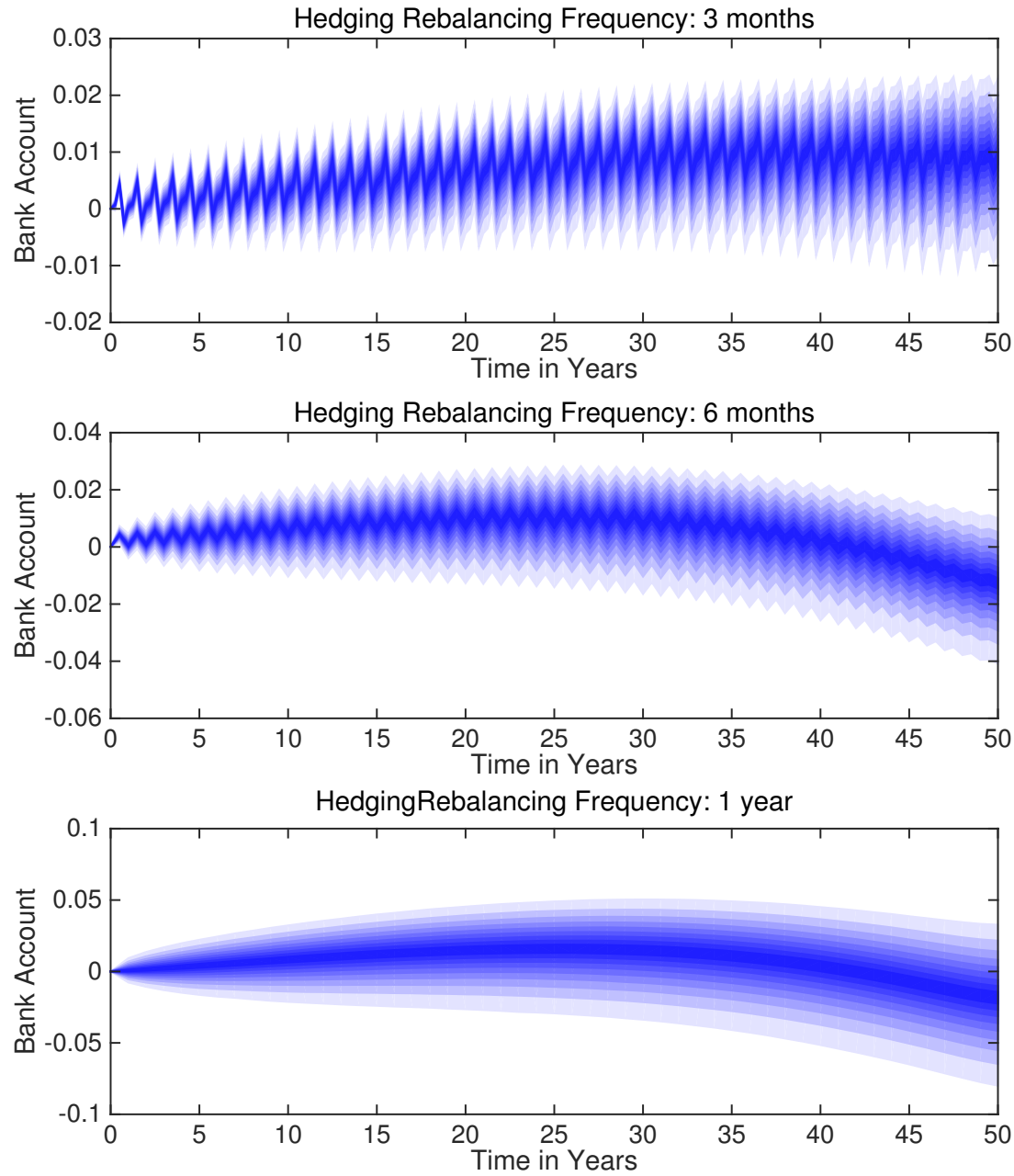

Figure 5. Percentiles of the bank account with basis risk and under different assumptions on the hedging rebalancing frequency. 


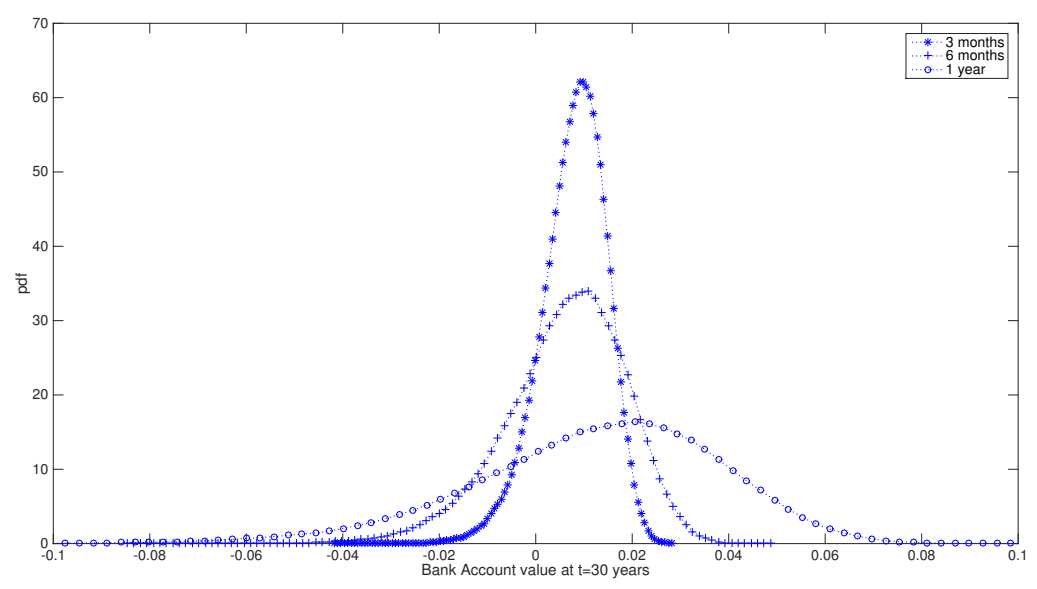

Figure 6. Distribution of the value of the bank account, under the assumption of basis risk, at $\mathrm{t}=30$ years for different rebalancing frequencies.

the bank account. A similar information is conveyed by Figure 6, that shows that the distributions of the bank account are no more centered at zero and present a high degree of asymmetry with long left tails. Table 7 reports the first two moments of the hedging error of the Delta-Gamma-Theta strategy, at time $t=30$ years, for each rebalancing frequency. Its mean and standard deviation are higher than the corresponding moments computed without basis risk (see Table 3). By taking the difference between the average hedging error in the two cases, we can isolate the part of the hedging error caused by basis risk. For instance, fixing the rebalancing frequency at 3 months, the error due to basis risk is $0.00824=0.00897-0.00073$. We observe that, though the moments of the hedging error are decreasing in the rebalancing frequency, basis risk is the main determinant of the hedging error, while discrete time rebalancing has only relatively marginal effects.

\begin{tabular}{|c|c|c|c|c|c|c|c|}
\hline & 3 months & 6 months & 1 year & & 3 months & 6 months & 1 year \\
\hline Mean & 0.00897 & 0.01146 & 0.02362 & Mean & 2.08373 & 2.09597 & 2.11164 \\
\hline Std & 0.00522 & 0.00797 & 0.01657 & Std & 0.20691 & 0.20715 & 0.20850 \\
\hline
\end{tabular}

Table 7. Moments of the hedging error of the Delta-Gamma-Theta hedging strategy, with basis risk, under different rebalancing frequencies.
Table 8. Moments of the hedging error of the Delta-Gamma hedging strategy, with basis risk, under different rebalancing frequencies.

The longevity swap premiums, computed using the value-at-risk loading principle, are reported in Table 9 . They range now between 0.01226 and 0.04193 , and the corresponding percentage loading on the survival probabilities ranges from $0.12 \%$ to $0.41 \%$. A comparison with Table 5 highlights that 


\begin{tabular}{cccc}
\hline & 3 months & 6 months & 1 year \\
\hline$C_{0}$ & 0.01226 & 0.02010 & 0.04193 \\
$m$ & $0.12 \%$ & $0.20 \%$ & $0.41 \%$ \\
\hline
\end{tabular}

Table 9. Longevity Swap premiums and loadings equivalent to the $99.5 \%$ Value-atRisk of the Delta-Gamma-Theta Hedging strategy at $t=30$ years with basis risk.

\begin{tabular}{cccc}
\hline & 3 months & 6 months & 1 year \\
\hline$C_{0}$ & 1.48367 & 1.48762 & 1.49936 \\
$m$ & $14.42 \%$ & $14.46 \%$ & $14.57 \%$ \\
\hline
\end{tabular}

Table 10. Longevity Swap premiums and loadings equivalent to the $99.5 \%$ Value-atRisk of the Delta-Gamma Hedging strategy at $t=30$ years with basis risk.

when basis risk is present, the Delta-Gamma-Theta strategy yield higher costs $C_{0}$ and spreads $m$, as expected, but still remains fairly effective. If we compare the values in Tables 7 and 9 with those in Tables 8 and 10 , we immediately see that the Delta-Gamma strategy achieves an hedging error considerably higher than the Delta-Gamma-Theta strategy. Without basis risk the performances of the two strategies were similar but, if basis risk is present, this is no more the case. This result shows that even if hedging when basis risk is not negligible can be effective, the hedging strategy needs to be appropriately designed.

\section{Summary and conclusions}

This paper introduces a simple model for basis risk in longevity-linked securities, computes the static, customized, swap-based hedge for an annuity, and compares it with the dynamic, Delta-Gamma-Theta based hedge, achieved using indexed longevity bonds. All throughout, the paper assumes a non mean reverting CIR process for mortality intensity. In the theoretical part, we consider interest rate risk as well, while the empirical application focuses on longevity risk only. We show that, once the model is calibrated to a UK individual aged 65, if there is no basis risk the average hedging error of the dynamic hedge is moderate, and both its variance and the thickness of the tails of its distribution are decreasing with the rebalancing frequency. We compute the fee which makes the $99.5 \%$ quantile of the distribution of the dynamic hedging error at an horizon of 30 years equal to the cost of the static hedge. This stays between 0.01 and $0.06 \%$. When there is basis risk, modelled parsimoniously and consistently, the fee ranges between $0.12 \%$ to $0.41 \%$. The same does not hold with simpler Delta-Gamma strategies. We conclude that, while basis risk is indeed relevant for annuity provider's hedges, dynamic hedging strategies, such as Delta-Gamma-Theta, can still be fairly effective if they are calibrated and implemented appropriately, even when rebalancing occurs at low frequencies. So, even when basis risk is present, a priori, one could rely on dynamic hedging strategies, instead of structuring a fully customized OTC hedge.

This conclusion seems relevant for the development of longevity markets, 
as we show that standardized, index-based, products are effective hedges, if appropriately managed.

Robustness of our analysis with respect to different populations, annuity features, such as the presence of guarantees, different horizons, or longevity model specifications, are in the agenda for future research.

\section{References}

Barrieu, P., H. Bensusan, N. El Karoui, C. Hillairet, S. Loisel, C. Ravanelli, and Y. Salhi (2012). Understanding, modelling and managing longevity risk: key issues and main challenges. Scandinavian Actuarial Journal (3), 203-231.

Blake, D. and W. Burrows (2001). Survivor bonds: Helping to hedge mortality risk. Journal of Risk and Insurance, 339-348.

Cairns, A. J., K. Dowd, D. Blake, and G. D. Coughlan (2014). Longevity hedge effectiveness: A decomposition. Quantitative Finance 14(2), 217235.

Coughlan, G. D., M. Khalaf-Allah, Y. Ye, S. Kumar, A. J. Cairns, D. Blake, and K. Dowd (2011). Longevity hedging 101: A framework for longevity basis risk analysis and hedge effectiveness. North American Actuarial Journal 15(2), 150-176.

Cowley, A. and J. Cummins (2005). Securitization of life insurance assets and liabilities. The Journal of Risk and Insurance 72(2), 193-226.

Dahl, M., S. Glar, and T. Møller (2011). Mixed dynamic and static riskminimization with an application to survivor swaps. European Actuarial Journal 1(2), 233-260.

Dahl, M., M. Melchior, and T. Møller (2008). On systematic mortality risk and risk-minimization with survivor swaps. Scandinavian Actuarial Journal (2-3), 114-146.

Fung, M. C., K. Ignatieva, and M. Sherris (2014). Systematic mortality risk: An analysis of guaranteed lifetime withdrawal benefits in variable annuities. Insurance: Mathematics and Economics 58, $103-115$.

Haberman, S., V. Kaishev, P. Millossovich, and A. Villegas (2014). Longevity basis risk - a methodology for assessing basis risk. Report, Cass Business School and Hymans Robertson LLP.

International Monetary Fund (IMF) (2012). Global Financial Stability Report. (IMF April), 123-154. 
Jarrow, R. and S. Turnbull (1994). Delta, gamma and bucket hedging of interest rate derivatives. Applied Mathematical Finance 1, 21-48.

Li, J. and M. R. Hardy (2011). Measuring basis risk in longevity hedges. North American Actuarial Journal 15(2), 177-200.

Luciano, E., L. Regis, and E. Vigna (2012). Delta-gamma hedging of mortality and interest-rate risk. Insurance: Mathematics and Economics 50, 402-412.

Ngai, A. and M. Sherris (2011). Longevity risk management for life and variable annuities: The effectiveness of static hedging using longevity bonds and derivatives. Insurance: Mathematics and Economics 49, 100-114.

Wong, T. W., M. C. Chiu, and H. Y. Wong (2014). Time-consistent meanvariance hedging of longevity risk: Effect of cointegration. Insurance: Mathematics and Economics 56, 56-67. 